\title{
Periinterventionelle Therapie mit Thrombozyten- aggregationshemmern bei der Embolisation von nicht rupturierten zerebralen Aneurysmen
}

\author{
Stefan Wolff', Werner Weber ${ }^{2}$, Peter Berlit ${ }^{1}$, Dietmar Kühne ${ }^{2}$, Rolf R. Diehl ${ }^{1}$
}

\begin{abstract}
Zusammenfassung
Hintergrund: Als häufigste Komplikation der endovaskulären Behandlung zerebraler Aneurysmen treten thrombembolischischämische Ereignisse auf. Diese könnten möglicherweise durch eine präinterventionell beginnende Begleitmedikation mit einem Thrombozytenaggregationshemmer (TAH) reduziert werden.

Methodik: In einer Beobachtungsstudie wurden 128 Behandlungen nicht rupturierter Aneurysmen mit oder ohne präinterventionelle Anwendung eines TAH bezüglich der gerinnungsaktiven Medikation sowie der Häufigkeit thrombembolischer Komplikationen, Blutungen und stummer Hirnischämien analysiert.

Ergebnisse: Die Gesamthäufigkeit, -morbidität und -mortalität von prozedural verursachten Thrombembolien betrugen in dieser Studie 13,3\% bzw. 5,5\% bzw. 0\%. Häufigkeit und Mortalität waren in den beiden Behandlungsgruppen gleich. Bezüglich der Morbidität bestand ein statistischer Trend für ein besseres Outcome der vorbehandelten Patientengruppe $(1,7 \%$ bzw. $8,9 \% ; p=0,08)$. Zerebrale Blutungskomplikationen traten in den beiden Gruppen gleich häufig auf. Blutungen in anderen Körperregionen wurden nicht beobachtet.

Schlussfolgerung: Der Einsatz von TAH verursacht keine zusätzlichen Behandlungskomplikationen. Trotz eines statistischen Trends zugunsten einer geringeren Morbidität in der vorbehandelten Gruppe kann ein positiver Effekt der Prämedikation nicht nachgewiesen werden. Die Durchführung einer prospektiven Therapiestudie scheint aufgrund dieser Beobachtungsstudie unbedenklich.
\end{abstract}

Schlüsselwörter: Intrakranielle Aneurysmen · Coilokklusion · Komplikation · Thrombozytenaggregationshemmer • Schlaganfall

Clin Neuroradiol 2006;16:236-44

DOI $10.1007 / \mathrm{s} 00062-006-6028-7$

\section{Periinterventional Antiplatelet Therapy in Endovascular Occlusion of Unruptured Intracranial Aneurysms}

\begin{abstract}
Background: Thromboembolic events are a major complication associated with endovascular treatment of intracranial aneurysms. Their occurrence might be reduced by using antiplatelet agents as an accompanying pretreatment medication.

Methods: 128 endovascular occlusions of unruptured aneurysms with or without TAH pretreatment were analyzed in an observational study regarding antiplatelet therapy and frequency of thromboembolic complications, hemorrhages and silent microembolic strokes.

Results: The overall frequency, morbidity and mortality of thromboembolic events in this study were $13.3 \%, 5.5 \%$, and $0 \%$, respectively. Frequency and mortality were similar in the two treatment groups. In terms of morbidity there was a slightly (not significant) better outcome in the patient group exposed to an antiplatelet agent $(1.7 \%$ versus $8.9 \% ; p=$ 0.08). Cerebral hemorrhages were similar in the two groups. Bleedings in other parts of the body could not be observed.
\end{abstract}

\footnotetext{
${ }^{1}$ Klinik für Neurologie, Alfried-Krupp-Krankenhaus, Essen,

${ }^{2}$ Klinik für Neuroradiologie, Alfried-Krupp-Krankenhaus, Essen.
} 
Conclusion: Antiplatelet therapy prior to endovascular treatment of unruptured intracerebral aneurysms causes no additional bleeding complications. A significant positive effect of pretreatment could not be demonstrated. Implementation of a prospective study seems to be harmless.

Key Words: Intracranial aneurysms · Endovascular occlusion · Complication · Antiplatelet therapy · Stroke

\section{Einleitung}

Als häufigste Komplikation der endovaskulären Behandlung von zerebralen Aneurysmen treten thrombembolisch-ischämische Ereignisse auf. Die Häufigkeit dieser thrombembolischen Komplikationen wird in neueren Studien der letzten 5 Jahre mit 3,7-13,1\% angegeben [1-5]. In kernspintomographischen Studien konnten stumme Hirnischämien in bis zu $61 \%$ der Fälle nachgewiesen werden [6-8].

Prophylaktisch erfolgt intraprozedural der Einsatz von Antikoagulanzien (z.B. Heparin i.v.). Thrombozytenaggregationshemmer (TAH) werden bisher meist nur in besonderen Behandlungssituationen eingesetzt (z.B. bei breiter Kontaktfläche von Coilpacket und Blutstrom, bei Stentbehandlungen) [9, 10]. Über den Einsatz von TAH bei der endovaskulären Behandlung von zerebralen Aneurysmen gibt es bisher nur wenige Daten. In zwei Studien, die einen hohen Anteil an stummen Hirninfarkten nach Coilembolisationen zeigten, wird der mögliche primärprophylaktische Einsatz von TAH bereits vor Behandlungsbeginn diskutiert $[7,8]$.

In dieser retrospektiven Analyse soll untersucht werden, ob durch eine bereits präinterventionell beginnende Gabe von TAH bei der Behandlung nicht rupturierter Aneurysmen positive Effekte bezüglich Gesamthäufigkeit, Morbidität und Mortalität von Thrombembolien auftreten oder ob negative Effekte durch eventuelle Blutungskomplikationen überwiegen. Dazu wurden die gerinnungsaktive Medikation von konsekutiv über 2 Jahre behandelten Aneurysmen analysiert und zwei Behandlungsgruppen mit und ohne TAH miteinander verglichen.

\section{Patienten und Methodik}

\section{Patienten und Aneurysmen}

Insgesamt wurden 215 konsekutive Aneurysmaprozeduren aus den Jahren 2002 und 2003 retrospektiv ausgewertet. Folgende Einschlusskriterien mussten die Aneurysmen für diese Studie erfüllen:
- nicht rupturiert,

- intrakranielle Lokalisation,

- alleinige Behandlung mit Platincoils.

Von der Analyse wurden 46 rupturierte Aneurysmen und 41 weitere Aneurysmen mit anderen Therapieverfahren (Stent, Onyx ${ }^{\circledR}$, Verschluss des Trägergefäßes) ausgeschlossen. Insgesamt wurden 128 Aneurysmen von 103 Patienten in diese Analyse einbezogen.

Zur Bestimmung der Aneurysmadimensionen wurde der Durchmesser des Halses und der größten Distanz des Fundus gemessen. Als Referenzweite diente das Gefäßkaliber der Arteria carotis interna im petrösen Anteil (mit $5 \mathrm{~mm}$ ) sowie das der Arteria vertebralis im V4-Abschnitt (mit $4 \mathrm{~mm}$ ). In Anlehnung an die Kriterien der International Study of Unruptured Intracranial Aneurysms zur Aneurysmagröße erfolgte eine Einteilung in kleine $(<7 \mathrm{~mm})$, mittelgroße $(7-12 \mathrm{~mm})$, große (13-24 mm) und sehr große Aneurysmen $(\geq 25 \mathrm{~mm})$ [11].

Der Verschlussgrad der Aneurysmen wurde nach den von Roy et al. beschriebenen Kriterien in drei Kategorien eingeteilt [1]. Ein kompletter Verschluss besteht demnach bei dichter Coilfüllung des Aneurysmahal-ses und -sacks in allen Projektionsrichtungen („,complete“). In die zweite Kategorie fallen Aneurysmen mit einem dicht coilgefüllten Sack, bei denen ein minimaler Resteinstrom ( $<2 \mathrm{~mm}$ ) sichtbar ist (,residual neck"). Für die dritte Kategorie bleiben die Aneurysmen mit lockerer Coilfüllung des Sacks oder verbliebenen Resteinstrom von mehr als $2 \mathrm{~mm}$ (,residual aneurysm“).

\section{Periinterventionelle Medikation und neurologische Untersuchung}

Die Unterteilung der insgesamt 128 endovaskulären Prozeduren in zwei Behandlungsgruppen erfolgte nach Analyse der präinterventionellen thrombozytenaggregationshemmenden Medikation.

In die Gruppe mit medikamentöser Vorbehandlung fielen alle Patienten, die mindestens 1 Tag vor der In- 
tervention (d.h. am Tag vor und am Tag der Intervention) einen TAH (z.B. Acetylsalicylsäure [ASS] oder Clopidogrel) erhalten hatten. Es wurden die üblichen Dosierungen von $100 \mathrm{mg}$ ASS oder 75 mg Clopidogrel verwendet. Die teilweise kurze Einnahmedauer der Medikation war für einen thrombozytenaggregationshemmenden Effekt ausreichend [12]. Wegen einer ursprünglich geplanten, dann aber nicht durchgeführten stentassistierten Behandlung erhielten einige Patienten eine kombinierte Therapie mit einer Startdosis von $500 \mathrm{mg}$ ASS und $300 \mathrm{mg}$ Clopidogrel, die mit einer Erhaltungsdosis von $100 \mathrm{bzw} .75 \mathrm{mg}$ fortgesetzt wurde. Bei den meisten Patienten erfolgte die Einnahme des TAH aufgrund anderer Erkrankungen und nicht gezielt als Prämedikation für den interventionellen Eingriff.

In die Gruppe ohne medikamentöse Vorbehandlung fielen alle Patienten, die mindestens 10 Tage vor der Intervention keinen TAH erhalten hatten. Dieser Zeitraum richtete sich nach der ungefähren Lebensdauer von Thrombozyten [12]. Es gab keinen Patien-ten, der einen TAH innerhalb dieser 10-Tage-Frist absetzte, so dass die Zuordnung zu den beiden Behandlungsgruppen immer eindeutig vorgenommen werden konnte.

Eine ausführliche neurologische Untersuchung wurde von neurologischen Fachärzten präinterventionell, unmittelbar nach der Intervention und bei Entlassung durchgeführt. Bei Fällen mit prozedurabhängigen neuen zerebralen Komplikationen erfolgte analog zu anderen Studien [3,13] die Klassifizierung der Schweregrade neurologischer Defizite mit der Glasgow Outcome Scale (GOS) [14]. Eine Unterteilung in ischämische und hämorrhagische Komplikationen erfolgte anhand radiologischer Kriterien. Prozedurabhängige neurologische Defizite wurden wie folgt bezeichnet: transiente Defizite, die sich innerhalb von 7 Tagen zurückbildeten; persistierende Defizite, die länger als 7 Tage andauerten. Dementsprechend war die prozedurabhängige neurologische Morbidität definiert als neurologische Defizite, die länger als 7 Tage andauerten und auf die neuroradiologische Prozedur zurückzuführen waren [5].

\section{Neuroradiologische Intervention}

Die endovaskulären Behandlungen wurden von drei erfahrenen, interventionell tätigen Neuroradiologen am Alfried-Krupp-Krankenhaus in Essen jeweils unter Intubationsnarkose durchgeführt.

Die digitalen Subtraktionsangiographien wurden an einem Zwei-Ebenen-Gerät (Neurostar, Siemens, Erlangen) angefertigt. Es kamen handelsübliche 6-French-
Führungskatheter und Mikrokatheter verschiedener Hersteller zum Einsatz. Zur Embolisation wurden sowohl unbeschichtete (sog. „bare coils“) als auch faserbesetzte Platinmikrospiralen eingesetzt.

\section{MRT}

Kernspintomographische Untersuchungen wurden mit einem 1,5-Tesla-Gerät (Magnetom Sonata, Siemens Medical Solutions, Erlangen, Deutschland) für das postinterventionelle Schlaganfallscreening durchgeführt. Folgende Sequenzen wurden benutzt: T2/TSE (Repetitionszeit 4150 ms, Echozeit 120 ms, Turbofaktor 15, Schichtdicke $5 \mathrm{~mm}$, Dauer 3,37 min), EPI-Sequenz (Repetitionszeit $2900 \mathrm{~ms}$, Echozeit $83 \mathrm{~ms}$, Turbofaktor 128, Schichtdicke $5 \mathrm{~mm}$, Dauer 1,07 min). Es wurde eine zirkulär polarisierte Kopfspule verwendet. Die Untersuchung fand immer 1-3 Tage nach der endovaskulären Behandlung statt. Unabhängig von der ursprünglichen Befundung erfolgte eine nachträgliche geblindete Auswertung durch einen neuroradiologischen Facharzt. Die MRT-Bilder lagen als digitale Bilddateien an speziellen PACS-(Picture Archiving and Communication System-)Work-stations vor.

\section{Statistik}

Der statistische Vergleich von Häufigkeiten erfolgte mit dem $\chi^{2}$-Test oder dem exakten Fisher-Test. Irrtumswahrscheinlichkeiten von $\mathrm{p}<0,05$ wurden als statistisch signifikant bezeichnet. Parametrische Daten werden als Mittelwert mit Standardabweichung oder mit Spannweite angegeben.

\section{Ergebnisse \\ Epidemiologie}

Von den mit einem TAH vorbehandelten Aneurysmen waren 26 klein $(<7 \mathrm{~mm}), 28$ mittelgroß $(7-12 \mathrm{~mm})$, vier groß (13-24 mm) und zwei sehr groß ( $\geq 25 \mathrm{~mm}$ ). Für die nicht vorbehandelten Aneurysmen betrugen diese Zahlen 30 bzw. 33 bzw. vier bzw. eins. Insgesamt waren $56(43,8 \%)$ von allen Aneurysmen klein, 61 (47,7\%) mittelgroß, acht $(6,2 \%)$ groß und drei $(2,3 \%)$ sehr groß. Eine Zusammenfassung der demographischen und morphologischen Daten der Aneurysmen, stratifiziert nach der Vormedikation mit einem TAH, findet sich in Tabelle 1.

Die Embolisation von 60 Aneurysmen wurde unter einer Medikation mit einem TAH durchgeführt. Die Einnahme des TAH bei 51 Prozeduren erfolgte dabei aus nichtneuroradiologischen Gründen (ASS 45 
Prozeduren, Clopidogrel [Plavix ${ }^{\circledR}$ ] sechs Prozeduren). Bei neun Prozeduren war die Kombination der beiden TAH als Stentprämedikation eingesetzt worden. 68 Aneurysmen wurden ohne gerinnungsaktive Vorbehandlung therapiert.

Bei $85 \%$ der Interventionen (109/128) waren die Patienten während des Eingriffs durch ein- bzw. mehrmalige intravenöse Bolusgaben von unfraktioniertem Heparin in PTT-wirksamen Dosen antikoaguliert. Die Übrigen erhielten keine dementsprechende Begleitmedikation $(\mathrm{n}=19) .29$ der vorbehandelten und 37 der nicht vorbehandelten Patienten bekamen nach Abschluss der Intervention zusätzlich einen einmaligen Bolus von 500 mg Aspisol ${ }^{\circledR}$ i.v. (Acetylsalicylsäure). Diese Komedikation wurde von den interventionellen Neuroradiologen nach Maßgabe der aktuellen Behandlungssituation eingesetzt (z.B. bei größerer Grenzfläche zwischen Coilpacket und Trägergefäß).

\section{Prozedurale Komplikationsrate, Morbidität, Mortalität}

Insgesamt traten bei 22 von 128 Prozeduren (17,2\%) prozedurabhängige zerebrale Komplikationen auf, die klinisch auffällig waren. Dabei entfielen 17 auf thrombembolisch-ischämische Ereignisse und fünf auf hämorrhagische Komplikationen. Bei 17 Komplikationen traten nur minimale oder leichte (GOS IV-V), bei vier mittelschwe-re (GOS III) und bei einer schwere (GOS II) neurologische Defizite auf.

Die Gesamtmorbidität, also der Anteil der Prozeduren mit postinterventionell länger als 7 Tagen persistierenden neurologischen Defiziten, betrug 5,5\% (7/128). Diese Defizite waren ohne Ausnahme durch thrombembolisch-ischämische Komplikationen aufgetreten. Ein Patient verstarb im Verlauf wegen einer ausgedehnten Subarachnoidalblutung (SAB) nach iatrogener Aneurysmaruptur, so dass die Gesamtmortalität $0,8 \%$ betrug.

\section{Thrombembolische Ereignisse}

ThrombembolischeKomplikationen traten bei insgesamt 17 (13,3\%) von 128 Prozeduren auf. Zehn davon waren transiente neurologische Defizite. Persistierende neurologische cranial aneurysms.
Defizite waren nach sieben Prozeduren nachweisbar, so dass die thrombembolische Morbidität 5,5\% betrug. Todesfälle gab es nicht.

In der Gruppe der mit einem TAH vorbehandelten Patienten $(\mathrm{n}=60)$ traten bei sieben Prozeduren $(11,7 \%)$ postinterventionell neue thrombembolische bedingte Defizite auf. Diese entsprachen initial ohne Ausnahme einem GOS-Schweregrad von IV. Bei sechs Patienten bildeten sich die Symptome innerhalb von 7 Tagen vollständig zurück. Ein Patient musste wegen einer leichtgradigen Armparese krankengymnastisch ambulant weiterbehandelt werden. Die Morbidität für thrombembolisch-ischämische Komplikationen unter Vorbehandlung mit einem TAH betrug demnach 1,7\% (Tabelle 2).

Bei den nicht vorbehandelten Patienten $(n=68)$ wurden unmittelbar nach den Interventionen in zehn Fällen $(14,7 \%)$ neue neurologische Defizite aufgrund von Thrombembolien nachgewiesen. In sechs Fällen traten leichte (GOS IV), bei vier mittelschwere Symptome (GOS III) auf. Bei sechs Patienten hielten die Symptome länger als 7 Tage an und bedurften einer stationären Rehabilitationsbehandlung. Die Morbidität für thrombembolisch-ischämische Komplikationen bei Patienten dieser Gruppe betrug demnach $8,9 \%$.

In Bezug auf die prozedurale Komplikationsrate durch thrombembolische Ereignisse ergab sich zwischen

Tabelle 1. Demographische und morphologische Daten der 128 intrakraniellen, nicht rupturierten, endovaskulär behandelten Aneurysmen.

Table 1. Demographic and morphological data of 128 endovascularly treated unruptured intra-

\begin{tabular}{lll}
\hline & TAH $(\mathbf{n}=\mathbf{6 0})$ & Kein TAH $(\mathbf{n}=\mathbf{6 8})$ \\
\hline Patientenzahl (m: w) & $47(9: 38)$ & $56(20: 36)$ \\
Alter in Jahren (Mittelwert mit Standardabweichung) & $54,4 \pm 12,2$ & $54,3 \pm 8,5$ \\
Aneurysmagröße in mm (Mittelwert mit Spannweite) & & \\
$\quad$ Hals & $4,5(1-10)$ & $4,5(2-10)$ \\
$\quad$ Fundus & $7,9(2-25)$ & $7,7(2-25)$ \\
Verschlussgrad (nach [1]) & & \\
$\quad$ Complete & $37(61,7 \%)$ & $46(67,6 \%)$ \\
$\quad$ Residual neck & $11(18,3 \%)$ & $12(17,6 \%)$ \\
$\quad$ Residual aneurysm & $12(20,0 \%)$ & $10(14,7 \%)$ \\
Lokalisation der Aneurysmen & & \\
ICA & $20(33,3 \%)$ & $23(33,8 \%)$ \\
MCA & $18(30,0 \%)$ & $19(27,9 \%)$ \\
AcomA & $11(18,3 \%)$ & $9(13,2 \%)$ \\
BA & $6(10,0 \%)$ & $8(11,8 \%)$ \\
Andere & $5(8,3 \%)$ & $9(13,2 \%)$ \\
\hline
\end{tabular}

AcomA: Arteria communicans anterior; BA: Arteria basilaris; ICA: Arteria carotis interna; MCA: Arteria cerebri media; m: männlich; TAH: Thrombozytenaggregationshemmer; w: weiblich 
Tabelle 2. Thrombembolisch-ischämische Komplikationen in den beiden Behandlungsgruppen unmittelbar postinterventionell und bei Entlassung bzw. am 7. postinterventionellen Tag (mit Berücksichtigung der klinischen Relevanz durch Unterteilung in Glasgow-Coma-Scale[GOS-]Schweregrade).

Table 2. Thromboembolic ischemic events in both treatment groups immediately after the intervention and after 7 days or at discharge, respectively (the clinical relevance is scored by Glasgow Coma Scale [GOS]).

\begin{tabular}{lllll}
\hline & TAH $(\mathbf{n}=\mathbf{6 0})$ & \multicolumn{3}{l}{ Kein TAH $(\mathbf{n}=\mathbf{6 8})$} \\
\hline Ischämien & $7(11,7 \%)$ & & $10(14,7 \%)$ \\
\hline & Postinterventionell & Entlassung & Postinterventionell & Entlassung \\
\hline GOS V & - & 6 & - & 4 \\
GOS IV & 7 & 1 & 6 & 5 \\
GOS III & - & - & 4 & 1 \\
GOS II & - & - & - & - \\
GOS I & - & - & - & - \\
\hline
\end{tabular}

In der vorbehandelten Patientengruppe traten zwei Hirnblutungen auf $(3,3 \%)$. Es handelte sich um eine nahezu asymptomatische SAB bei iatrogener Aneurysmaruptur und einen eingebluteten Mediateilinfarkt mit transientem Gesichtsfeldausfall (Prämedikation jeweils mit ASS).

In der nicht vorbehandelten Gruppe kam es zu drei Subarachnoidalblutungen $(4,4 \%)$. Ursächlich dafür waren zwei iatrogene Aneurysmarupturen und ein Wandeinriss des Trägergefäßes infolge einer Perforation mit dem Führungsdraht. Davon verlief eine Aneurysmaruptur tödlich (keine Medikation während Interven-

den Behandlungsgruppen kein statistisch signifikanter Unterschied $(11,7 \%$ vs. $14,7 \%$; $=0,6)$. Auch der Unterschied bezüglich der Morbidität (1,7\% vs. 8,9\%; $\mathrm{p}=$ $0,08)$ war nicht signifikant. Die Mortalitätsrate betrug in beiden Gruppen $0 \%$.

\section{Hirnblutungen}

Zerebrale hämorrhagische Komplikationen traten bei insgesamt fünf $(3,9 \%)$ von 128 Prozeduren auf. Bei vier Patienten traten nur minimale bis leichte neurologische Defizite auf, die sich komplett zurückbildeten. Die Morbidität für hämorrhagische Komplikationen betrug daher 0\%. Ein Patient mit einem initial schweren neurologischen Defizit verstarb (Mortalität $0,8 \%)$.

Tabelle 3. Hämorrhagische Komplikationen in den beiden Behandlungsgruppen unmittelbar postinterventionell und bei Entlassung bzw. am 7. postinterventionellen Tag (mit Berücksichtigung der klinischen Relevanz durch Unterteilung in Glasgow-Coma-Scale-[GOS-]Schweregrade).

Table 3. Hemorrhagic complications in both treatment groups immediately after the intervention and after 7 days or at discharge, respectively (the clinical relevance is scored by Glasgow Coma Scale [GOS]).

\begin{tabular}{lllll}
\hline & TAH $(\mathbf{n}=\mathbf{6 0})$ & \multicolumn{3}{l}{ Kein TAH $(\mathbf{n}=\mathbf{6 8})$} \\
\hline Blutungen & $2(3,3 \%)$ & \multicolumn{3}{l}{$3(4,4 \%)$} \\
\hline & Postinterventionell & Entlassung & Postinterventionell & Entlassung \\
\hline GOS V & 1 & 2 & 1 & 2 \\
GOS IV & 1 & - & 1 & - \\
GOS III & - & - & - & - \\
GOS II & - & - & 1 & - \\
GOS I & - & - & - & 1 \\
\hline
\end{tabular}

tion). Bei den beiden anderen Ereignissen traten jeweils nur transiente klinische Symptome auf (einmal ASS und einmal keine Medikation während Intervention).

Der Unterschied der Blutungshäufigkeit in den Behandlungsgruppen war nicht signifikant. Klinisch relevante Blutungen in anderen Körperregionen traten nicht auf (Tabelle 3).

\section{Stumme Thrombembolien im MRT}

Von der Gruppe der klinisch asymptomatisch verlaufenden Behandlungsprozeduren erhielten 60 Patienten zur Befundkontrolle eine postinterventionelle zerebrale Kernspintomographie. In der mit einem TAH vorbehandelten Patientengruppe waren klinisch stumme, diffusionsgewichtet nachweisbare Ischämien in $14,8 \%$ (vier von 27), in der nicht vorbehandelten Gruppe in 21,2\% der Fälle (sieben von 33) nachweisbar. Der Unterschied war nicht signifikant. Ein Beispiel für stumme Hirnischämien bei endovaskulärer Embolisation ist in Abbildung 1 dargestellt.

\section{Diskussion \\ Ergebnisinterpretation}

Bei einer prozeduralen Komplikationsrate von $17,2 \%$ für alle Aneurysmen zeigt die weitere Auswertung eine deutlich niedrigere Morbidität von $5,5 \%$ und eine Mortalität von $0,8 \%$. In der jüngeren Literatur wer- 
den prozedurale Komplikationsraten für nicht rupturierte Aneurysmen mit 6,9-10,9\% angegeben [1, 4, 5]. $\mathrm{Ng}$ et al. fanden mit $21 \%$ eine höhere Rate, die sich allerdings auf rupturierte und nicht rupturierte Aneurysmen bezog [3]. Die hier angegebene prozedurale Komplikationsrate gibt die Häufigkeit aller post-interventionell neu aufgetretenen klinischen Ereignisse wieder. Die Ursache für die höhere Rate im Literaturvergleich ist am ehesten in der Erfassung auch flüchtiger Komplikationen, z.B. transienter Hemiparesen oder eines Meningismus bei behandlungsbedingter Aneurysmaruptur, zu sehen. Die Morbiditäts- und Mortalitätsraten liegen im Bereich der Literaturangaben von 1,4-5,5\%, bzw. 0,0-1,4\% [1,3-5].

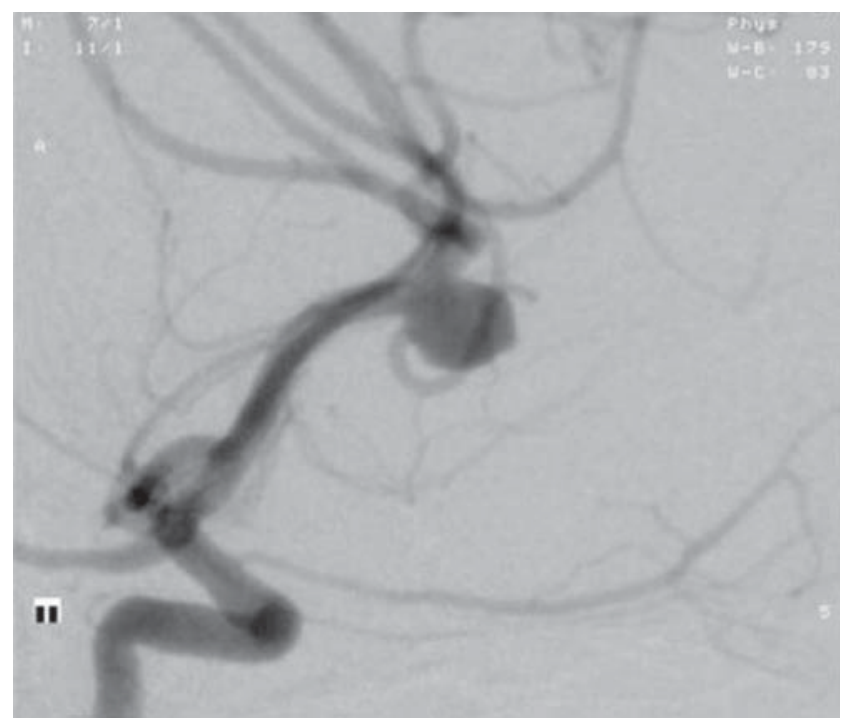

Abbildung 1a - Figure 1a

\begin{abstract}
Abbildungen 1a bis 1c. Stumme Hirnischämien bei Coilembolisation eines asymptomatischen nicht rupturierten Aneurysmas der Arteria cerebri media links bei einer 57-jährigen Patientin ohne Vorbehandlung mit einem TAH: präinterventioneller Gefäßstatus, Aneurysmahals $5 \mathrm{~mm}$, -fundus $7 \mathrm{~mm}$ (a). Gefäßstatus nach Embolisation mit Protrusion einer Coilschlinge in das Gefäßlumen als potentielle Emboliequelle (b). Zwei punktförmige frische Hirnischämien in postinterventioneller Kernspintomographie (diffusionsgewichtete Aufnahmen, c).

Figures 1a to 1c. Silent infarctions after coil embolization of an asymptomatic unruptured aneurysm of the left middle cerebral artery in a 57-year-old woman without antiplateletpretreatment. Preinterventional angiogram showing the aneurysm with diameters of $5 \mathrm{~mm}$ (neck) and $7 \mathrm{~mm}$ (fundus), respectively (a). Postinterventional angiogram showing the protrusion of a coil loop into the vessel lumen as a potential source of embolism (b). Two punctual fresh infarctions postinterventionally demonstrated by diffusion-weighted MRI (c).
\end{abstract}

Thrombembolische Komplikationen sind mit einem Anteil von 13,3\% aller Prozeduren in dieser Serie am häufigsten. Die thrombembolische Morbidität liegt bei $5,5 \%$. Dies ist vergleichbar mit Studienergebnissen, in denen die thrombembolische Morbidität nach der Behandlung nicht rupturierter Aneurysmen zwischen 1,4\% und $5,0 \%$ betrug $[5,8,15]$. Interessant ist, dass die $\mathrm{Ge}$ samtmorbidität ausschließlich durch thrombembolische Komplikationen und die Gesamtmortalität ausschließlich durch hämorrhagische Komplikationen hervorgerufen werden. Dies stimmt mit der Beobachtung von Park et al. überein, wonach intraprozedurale Aneurysmarupturen meist entweder ein gutes Outcome ohne klinisches Defizit oder ein tödliches Outcome haben [5].

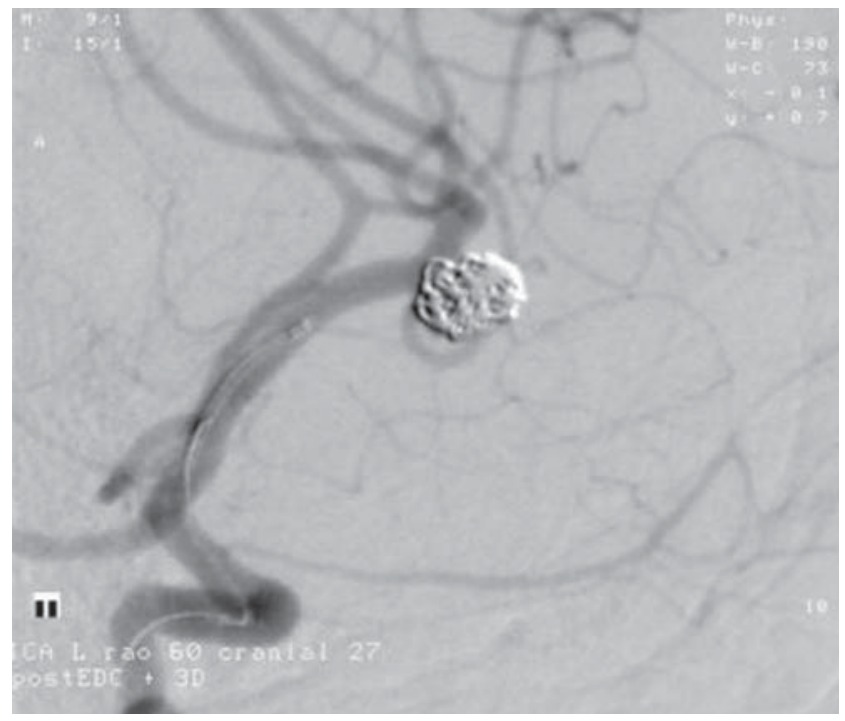

Abbildung $1 \mathrm{~b}$ - Figure $1 \mathrm{~b}$

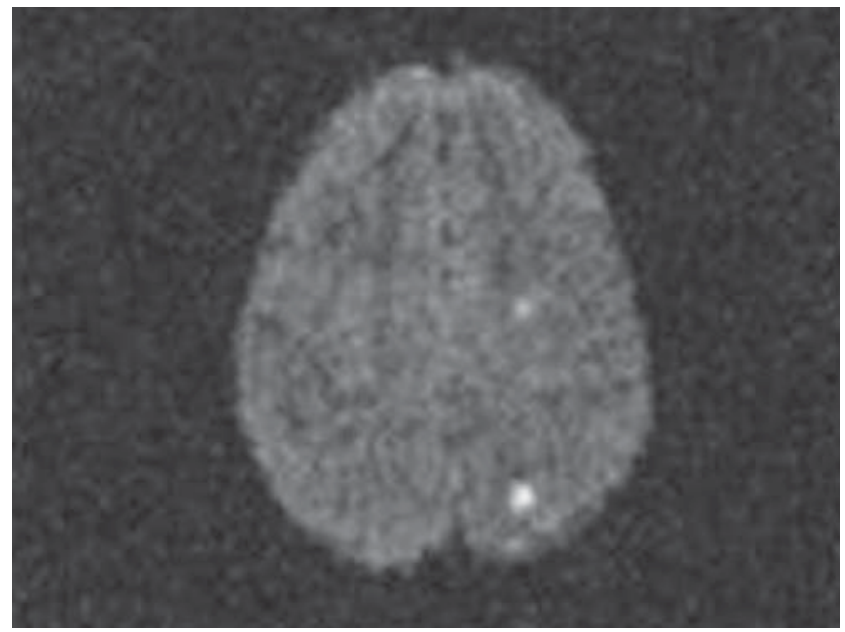

Abbildung 1c - Figure 1c 
Die thrombembolischen Komplikationsraten der beiden Behandlungsgruppen unterscheiden sich nicht signifikant (11,7\% vs. $14,7 \%)$. Der klinische Verlauf ist in der Regel gutartig. Insbesondere in der Gruppe der mit einem TAH vorbehandelten Patienten beträgt die Morbidität nur 1,7\%. Das klinische Outcome der nicht vorbehandelten Patienten stellte sich mit sechs persistierenden klinischen Defiziten bei Entlassung ungünstiger dar. Die Morbidität beträgt in dieser Gruppe 8,9\%. Der Unterschied ist mit einem Signifikanzniveau von $\mathrm{p}=0,08$ nicht signifikant, kann aber als statistischer Trend für ein besseres klinisches Outcome der vorbehandelten Patientengruppe gewertet werden. Die thrombembolische Mortalitätsrate beider Gruppen beträgt $0 \%$.

Klinisch stumme Hirnembolien finden sich $\mathrm{zu}$ einem geringeren Anteil in der vorbehandelten Patientengruppe (14,8\% vs. $21,2 \%)$. Dieses Ergebnis war statistisch nicht signifikant $(p \sim 0,5)$. Eine mögliche günstige Beeinflussung von thrombembolischen Komplikationen durch einen TAH lässt sich hieraus daher nicht ableiten. Hinderlich für eine exakte Auswertung war die Tatsache, dass postinterventionelle Kernspintomographien des Hirnschädels nur bei $56,6 \%$ aller asymptomatischen Patienten vorlagen $(52,9 \%$ in der vorbehandelten, $60 \%$ in der nicht vorbehandelten Gruppe). In der Literatur werden die Häufigkeiten von klinisch stummen Hirninfarkten mit 36\% [8] bzw. 61\% [7] angegeben. Die Behandlung großer Aneurysmen und die ballonassistierte Technik werden als Prädiktoren für stumme Embolien genannt. In beiden Studien waren viele Aneurysmen ballonassistiert behandelt worden (Anteil: 49\% bzw. $36 \%$ ). Bei den Embolisationen dieser Analyse wurde dagegen keine ballonunterstützte Technik verwendet. Außerdem war der Anteil großer und sehr großer Aneurysmen mit 8,5\% niedrig. Insgesamt war demnach eine geringere Rate an stummen Hirnembolien in dieser Analyse zu erwarten.

Die periprozedurale Blutungshäufigkeit ist in beiden Patientengruppen vergleichbar (3,3\% bzw. 4,4\%) und entspricht den niedrigen Blutungsraten anderer Studien mit nicht rupturierten Aneurysmen von $0,0-4 \%$ $[1,3-6,16]$. Da in der periprozeduralen Blutungshäufigkeit eine hämorrhagisch transformierte Hirnischämie enthalten ist, beträgt die intraprozedurale Rupturrate für alle Aneurysmen sogar nur 3,1\%. Insgesamt ist der klinische Verlauf dieser Hirnblutungen äußerst gutartig. Insbesondere in der mit einem $\mathrm{TAH}$ vorbehandelten
Gruppe gibt es keine persistierenden klinisch-neurologischen Defizite (Morbidität 0\%) und keine Todesfälle (Mortalität 0\%). Auch in der Patientengruppe ohne TAH-Vorbehandlung beträgt die Morbidität $0 \%$. Allerdings ist der einzige Todesfall in dieser Gruppe zu verzeichnen. Die ursächliche Aneurysmaruptur ereignete sich beim Versuch der ersten Coilablösung vom Mikrokatheter. Eine frühe Ruptur vor erster Coilablösung (und einen initial schlechten Hunt- und HessGrad) beschreiben Park et al. als prognostische Faktoren für ein fatales Outcome nach intraprozeduraler Aneurysmaruptur [5]. Insgesamt konnte kein signifikanter Unterschied der beiden Patientengruppen bezüglich hämorrhagischer Komplikationen beobachtet werden.

\section{Limitationen der Arbeit}

Die intraprozedural verabreichte gerinnungsaktive Medikation war uneinheitlich und blieb bei der Auswertung dieser Studie unberücksichtigt. Insbesondere die einmalige Gabe eines TAH (500 mg ASS i.v.) bei etwa der Hälfte der nicht vorbehandelten Patienten war für die Datenbeurteilung problematisch. Vor allem für die Auswertung der hämorrhagischen Komplikationen spielt die einmalige intraprozedurale Gabe eines TAH in dieser Gruppe eine Rolle. Unter diesen Bedingungen würde man verhältnismäßig zu viele bzw. schwerer verlaufende Blutungen erwarten. Dies ist mit der einen tödlich verlaufenden Hirnblutung in der nicht vorbehandelten Patientengruppe auch eingetreten. Allerdings war genau in diesem Fall kein zusätzlicher TAH verabreicht worden, da die Blutung bereits bei der ersten Coilablösung aufgetreten war. Von den anderen beiden - blande verlaufenden - Blutungen in dieser Gruppe war in dem einen Fall eine einmalige Gabe eines TAH erfolgt, in dem anderen Fall nicht. Als intraprozedurale Komedikation blieb auch der Einsatz von intravenös verabreichtem Heparin für die Auswertung der Studie unberücksichtigt.

Als Prämedikation waren ASS, Clopidogrel und die Kombination von beiden eingesetzt. Andere TAH, wie z.B. Ticlopidin, wurden nicht verwendet. Eine Subgruppenanalyse für die verschiedenen Medikamente wurde wegen der kleinen Clopidogrel- und Kombinationsgruppen nicht durchgeführt. Es bleibt daher ungeklärt, ob es durch die Art des TAH zu Unterschieden im klinischen Outcome der vorbehandelten Patienten kommen könnte. Abweichende Wirksamkeiten der einzelnen TAH bei der Sekundärprä- 
vention von Schlaganfällen und bei der Hemmung von Stentthrombosen sind bekannt [17-19]. Man muss daher auch Unterschiede beim Einsatz als Prämedikation vor endovaskulären Behandlungen von Aneurysmen vermuten.

\section{Klinische Relevanz der Ergebnisse}

Insgesamt waren negative Effekte durch Anwendung der thrombozytenaggregationshemmenden Prämedikation nicht festzustellen. Die Blutungshäufigkeit war in beiden Patientengruppen gleich, fast alle Hirnblutungen verliefen äußerst blande, und es trat nur eine tödliche Blutung auf, die in der nicht vorbehandelten Gruppe zu verzeichnen war. Es wurden also keine zusätzlichen Blutungsrisiken durch die TAH-Einnahme verursacht. Auf der anderen Seite war ein positiver Effekt nicht sicher nachzuweisen. Die Häufigkeit von klinischen thrombembolischen Komplikationen und von stummen Hirnischämien war in beiden Gruppen nicht signifikant unterschiedlich. Allerdings traten persistierende klinisch-neurologische Defizite aufgrund thrombembolischer Komplikationen in der vorbehandelten Gruppe tendenziell weniger auf. Auf Grundlage dieser Analyse ist der Einsatz von TAH bei der endovaskulären Embolisation von intrakraniellen nicht rupturierten Aneurysmen unbedenklich. Das Absetzen eines TAH vor einer endovaskulären Aneurysmabehandlung ist nach der vorliegenden Analyse nicht nötig.

Wegen des tendenziell besseren Outcomes der vorbehandelten Patientengruppe erscheint eine Therapieempfehlung für eine prophylaktische Gabe eines TAH gerechtfertigt zu sein. In Anlehnung an die Stentprämedikation sind eine Startdosis („loading dose") von $500 \mathrm{mg}$ ASS mindestens 1 Tag vor endovaskulärer Behandlung und eine nachfolgende Erhaltungsdosis von $100 \mathrm{mg}$ ASS pro Tag zu diskutieren. Eventuell wäre dann eine TAH-Gabe während der Behandlungssitzung überflüssig. Dieses Dosierungsschema wäre auch für eine prospektive Therapiestudie zu nutzen. Ob oder wie lange eine postinterventionelle Einnahme eines TAH sinnvoll ist, wurde nicht untersucht.

\section{Perspektiven}

In einer prospektiven Therapiestudie müsste die Wirksamkeit der einzelnen TAH oder deren Kombinationen als Prämedikation bei der Embolisation von Aneurysmen überprüft werden.

\section{Literatur}

1. Roy D, Milot G, Raymond J. Endovascular treatment of unruptured aneurysms. Stroke 2001;32:1998-2004.

2. Workman MJ, Cloft HJ, Tong FC, Dion JE, Jensen ME, Marx WF, Kallmes DF. Thrombus formation at the neck of cerebral aneurysms during treatment with Guglielmi detachable coils. AJNR Am J Neuroradiol 2002;23:1568-76.

3. $\mathrm{Ng} \mathrm{P}$, Khangure MS, Phatouros CC, Bynevelt M, ApSimon H, McAuliffe W. Endovascular treatment of intracranial aneurysms with Guglielmi detachable coils: analysis of midterm angiographic and clinical outcomes. Stroke 2002;33:210-7.

4. Gonzalez N, Murayama Y, Nien YL, Martin N, Frazee J, Duckwiler G, Jahan R, Gobin YP, Viñuela F. Treatment of unruptured aneurysms with GDCs: clinical experience with 247 aneurysms. AJNR Am J Neuroradiol 2004;25:577-83.

5. Park HK, Horowitz M, Jungreis C, Genevro J, Koebbe C, Levy E, Kassam A. Periprocedural morbidity and mortality associated with endovascular treatment of intracranial aneurysms. AJNR Am J Neuroradiol 2005; 26:506-14.

6. Grunwald IQ, Papanagiotou P, Politi M, Struffert T, Roth C, Reith W. Endovascular treatment of unruptured intracranial aneurysms: occurrence of thromboembolic events. Neurosurgery 2006;58,612-8; discussion 612-8.

7. Rordorf G, Bellon RJ, Budzik RE Jr, Farkas J, Reinking GF, Pergolizzi RS, Ezzeddine M, Norbash AM, Gonzalez RG, Putman CM. Silent thromboembolic events associated with the treatment of unruptured cerebral aneurysms by use of Guglielmi detachable coils: prospective study a pplying diffusion-weighted imaging. AJNR Am J Neuroradiol 2001; 22:5-10.

8. Soeda A, Sakai N, Sakai H, Iihara K, Yamada N, Imakita S, Nagata I. Thromboembolic events associated with Guglielmi detachable coil embolization of asymptomatic cerebral aneurysms: evaluation of 66 consecutive cases with use of diffusion-weighted MR imaging. AJNR Am J Neuroradiol 2003;24:127-32.

9. Qureshi AI, Luft AR, Sharma M, Guterman LR, Hopkins LN. Prevention and treatment of thromboembolic and ischemic complications associated with endovascular procedures: Part II - Clinical aspects and recommendations. Neurosurgery 2000;46:1360-75.

10. Kommission "Qualitätssicherung" der Deutschen Gesellschaft für Neuroradiologie. Endovaskuläre Therapie von Hirngefäßaneurysmen. Leitlinien der Deutschen Gesellschaft für Neuroradiologie, Online-Publikation, AWMF-Leitlinien-Register Nr. 047/015, www.uni-duesseldorf.de/ WWW/AWMF/ll/047-015.htm 2004.

11. ISUIA - International Study of Unruptured Intracranial Aneurysms Investigators. Unruptured intracranial aneurysms: natural history, clinical outcome, and risks of surgical and endovascular treatment. Lancet 2003;362:103-10.

12. Patrono C, Coller B, FitzGerald GA, Hirsh J, Roth G. Platelet-active drugs: the relationships among dose, effectiveness, and side effects: the Seventh ACCP Conference on Antithrombotic and Thrombolytic Therapy. Chest 2004;126234S-264S, Suppl 3.

13. Henkes H, Fischer S, Weber W, Miloslavski E, Felber S, Brew S, Kuehne D. Endovascular coil occlusion of 1811 intracranial aneurysms: early angiographic and clinical results. Neurosurgery 2004; 54:268-80.

14. Jennett B, Bond M. Assessment of outcome after severe brain damage. Lancet 1975;1:480-4.

15. Pelz DM, Lownie SP, Fox AJ. Thromboembolic events associated with the treatment of cerebral aneurysms with Guglielmi detachable coils. AJNR Am J Neuroradiol 1998;19:1541-7.

16. Kwon BJ, Han MH, Oh CW, Kim KH, Chang KH. Procedure-related haemorrhage in embolisation of intracranial aneurysms with Guglielmi detachable coils. Neuroradiology 2003;45:562-9. 
17. Jamieson DG, Parekh A, Ezekowitz MD. Review of antiplatelet therapy in secondary prevention of cerebrovascular events: a need for direct comparisons between antiplatelet agents. J Cardiovasc Pharmacol Ther 2005;10:153-61.

18. MATCH investigators. Aspirin and clopidogrel compared with clopidogrel alone after recent ischaemic stroke or transient ischaemic attack in high-risk patients (MATCH): randomised, double-blind, placebocontrolled trial. Lancet 2004;364:331-7.

19. Makkar RR, Eigler NL, Kaul S, Frimerman A, Nakamura M, Shah PK, Forrester JS, Herbert JM, Litvack F. Effects of clopidogrel, aspirin and combined therapy in a porcine ex vivo model of high-shear induced stent thrombosis. Eur Heart J 1998;19:1538-046.

\section{Korrespondenzanschrift}

Stefan Wolff

Psychiatriezentrum Münsingen

3110 Münsingen

Schweiz

Telefon (43/31) 7208-242, Fax -800

E-Mail: stefan.wolff@gef.be.ch 